Article

\title{
A Multi-Attribute Pearson's Picture Fuzzy Correlation-Based Decision-Making Method
}

\author{
Yun Jin ${ }^{1}$, Hecheng Wu ${ }^{1}$, Dechao Sun ${ }^{2, *}$, Shouzhen Zeng ${ }^{3,4, *} \mathbb{D}$, Dandan Luo ${ }^{4}$ and Bo Peng ${ }^{5}$ \\ 1 College of Economic and Management, Nanjing University of Aeronautics and Astronautics, Nanjing 211106, \\ China; yjin@nuaa.edu.cn (Y.J.); hcwu@nuaa.edu.cn (H.W.) \\ 2 College of Big Data and Software Engineering, Zhejiang Wanli University, Ningbo 315100, China \\ 3 College of Statistics and Mathematics, Zhejiang Gongshang University, Hangzhou 310018, China \\ 4 School of Business, Ningbo University, Ningbo 315211, China; ndluodandan@163.com \\ 5 School of Management, Nanchang University, Nanchang 330031, China; pb_1020@163.com \\ * Correspondence: sundechao123@163.com (D.S.); zszzx1@163.com (S.Z.)
}

Received: 1 August 2019; Accepted: 16 October 2019; Published: 21 October 2019

\begin{abstract}
As a generalization of several fuzzy tools, picture fuzzy sets (PFSs) hold a special ability to perfectly portray inherent uncertain and vague decision preferences. The intention of this paper is to present a Pearson's picture fuzzy correlation-based model for multi-attribute decision-making (MADM) analysis. To this end, we develop a new correlation coefficient for picture fuzzy sets, based on which a Pearson's picture fuzzy closeness index is introduced to simultaneously calculate the relative proximity to the positive ideal point and the relative distance from the negative ideal point. On the basis of the presented concepts, a Pearson's correlation-based model is further presented to address picture fuzzy MADM problems. Finally, an illustrative example is provided to examine the usefulness and feasibility of the proposed methodology.
\end{abstract}

Keywords: picture fuzzy sets; multi-attribute decision-making; correlation-based closeness index; Pearson's correlation

\section{Introduction}

The process of multi-attribute decision-making (MADM) includes sorting the potential candidate alternatives and seeking the best one according to the decision-makers' subjective evaluation of the attributes [1,2]. In the actual decision-making process, a complex and unpredictable environment always leads to more complex and difficult subjective assessment, which makes it difficult to measure or quantify alternatives accurately in the MADM process. Therefore, evaluation information based on real number expressions may not accurately reflect the inherent uncertainty, vagueness, or fuzziness of decision information. Thus, dealing with uncertainties to adapt to specific practical problems and improve the scientificity of decision-making results is an essential issue of MADM tasks in complex situations.

Rapoport et al. [3] pointed out that a crucial issue in the empirical measurement of membership functions is whether the degree of fuzziness is invariant under different scaling procedures. Smarandache [4] recently presented the neutrosophic set as a new stream to deal with uncertainty. Yager [5] focused on the Pythagorean complement. Using this complement, Yager [5] introduced a class of nonstandard Pythagorean fuzzy subsets whose membership grades are pairs $(a, b)$ satisfying the requirement $a^{2}+b^{2} \leq 1$. The theory of picture fuzzy sets (PFSs), initially introduced by Cuong and Kreinovich [6], was created as a highly efficient tool that enables DMs to handle uncertainty and ambiguity with ease. PFSs are characterized by the notion of degrees of membership, neutral membership, and nonmembership that satisfy the condition that the sum of the three degrees is no 
more than 1. Compared with the classic fuzzy set [7], intuitionistic fuzzy set (IFS) [8], and Pythagorean fuzzy set [5], the main feature of the PFS is that it is distinguished by the three different functions, which makes it able to fully consider degrees of acceptance, rejection conflict, and refusal; thus, it can adapt to a higher degree of uncertainty than others.

Owing to its superior ability to handle complex uncertainty, the theory of PFSs has been widely used in the MADM area up to now. For example, Cuong [9] studied a series of features of PFSs and introduced several kinds of distance measures between PFSs. Singh [10] presented a correlation coefficient for PFSs and applied it in MADM problems. Garg [11] developed several picture fuzzy aggregation operations and used them to solve MADM problems. Son [12] introduced some distance and similarity measures between PFSs and used them to handle clustering analysis problems. Wei [13] explored the aggregation methods for picture fuzzy sets. Wei [14] extended several Hamacher aggregation operators to PFS situations and explored their usefulness in the MADM field. Wei et al. [15] constructed a projection approach for PFSs and applied it to evaluate emerging technology companies. Wang et al. [16] gave a normalized picture fuzzy projection and used it to choose a construction project. Zhang et al. [17] studied a method based on PFSs for selecting offshore wind power stations. Jana et al. [18] established several Dombi aggregation methods for picture fuzzy decision analysis. Ashraf et al. [19] used t-norm and t-conorm to establish some picture fuzzy weighted geometric aggregation operators for MADM problems. Zeng et al. [20] investigated a novel picture fuzzy divergence measure and used it to solve a decision-making problem. More fuzzy MADM methods are be found in Liu and You [21] and Zhang et al. [22].

From the previous survey, we can see that a fast-growing number of valuable approaches and techniques have been presented to address picture fuzzy MADM problems. However, the majority of them are too complex to be applied. Specifically speaking, current methods often possess numerous parameters and symbols, which makes the application scope and adaptability of these methods unclear. This will cause great difficulties for decision-makers to utilize these methods in practical applications. To provide a simple and efficient method for MADM problems in a picture fuzzy environment, in this study we present a Pearson's correlation-based model for solving picture fuzzy MADM problems. The correlation coefficient (also named Pearson's correlation coefficient) is a scale-free measurement for the linear association between two variables and is a popular measurement tool in the decision-making area. In this study, we aimed to extend the classic correlation conception between real sets to the picture fuzzy situation and propose a new Pearson-type measure named the picture fuzzy correlation coefficient, whose prominent feature is that it lies in the interval $[-1,1]$. This is consistent with the range of classical correlation coefficients in statistics, whereas the current picture fuzzy correlation coefficients in the literature are bounded within unit interval [0,1]. Moreover, a novel concept of a correlation-based picture fuzzy closeness index is proposed to simultaneously calculate the relative proximity to the picture fuzzy positive ideal point and the relative distance from the picture fuzzy negative ideal one. A new Pearson's correlation-based model is then established to address MADM problems with picture fuzzy evaluation. A practical example concerning selecting emerging technology enterprises is given to verify the proposed methodology's practicality and effectiveness.

The rest of this paper is presented as follows: In Section 2, some fundamental concepts are briefly reviewed. A new correlation coefficient for PFSs and a corresponding weighted correlation coefficient are presented in Section 3. Subsequently, a Pearson's correlation-based model for MADM involving PFS information is constructed in Section 4, and a numerical example concerning emerging technology commercialization assessment is given to illustrate the effectiveness and feasibility of the proposed method in Section 5. Finally, the conclusions and future work are summarized in Section 6.

\section{Preliminaries}

In this section, some essential concepts relating to PFSs are briefly reviewed; these will be used in the rest of this work. 
Definition 1 [8]. An IFS A in $X=\left\{x_{1}, x_{2}, \ldots, x_{n}\right\}$ is defined as

$$
A=\left\{\left\langle x,\left(E_{A}(x), N_{A}(x)\right)\right\rangle \mid x \in X\right\}
$$

where the functions $0 \leq E_{A}(x) \leq 1$ and $0 \leq N_{A}(x) \leq 1$ are called the degrees of membership and nonmembership, respectively, with the condition $0 \leq E_{A}(x)+N_{A}(x) \leq 1$.

Definition 2 [6]. A picture fuzzy set (PFS) $P$ in $X=\left\{x_{1}, x_{2}, \ldots, x_{n}\right\}$ is defined as

$$
P=\left\{\left\langle x,\left(E_{P}(x), I_{P}(x), N_{P}(x)\right)\right\rangle \mid x \in X\right\}
$$

where $E_{P}(x) \in[0,1]$ is the degree of positive membership, $I_{P}(x) \in[0,1]$ is the degree of neutral membership, and $N_{P}(x) \in[0,1]$ denotes the degree of negative membership, satisfying the condition $0 \leq E_{P}(x)+I_{P}(x)+N_{P}(x) \leq 1, \forall x \in X$. The degree of refusal membership for each $x \in X$ is given as $T_{P}(x)=1-\left(E_{P}(x)+I_{P}(x)+N_{P}(x)\right)$.

Obviously, if $I_{P}(x)=0$, then the PFS reduces to Atanassov's IFS, which indicates that the IFS is a particular case of the PFS. For convenience of calculation, we use $p=(E, I, N)$ to denote a picture fuzzy value (PFV).

Definition 3. Let $p_{1}=\left(E_{1}, I_{1}, N_{1}\right)$ and $p_{2}=\left(E_{2}, I_{2}, N_{2}\right)$ be two picture fuzzy values (PFVs); some basic operational rules are defined as follows [13]:

(1) $\alpha_{1} \oplus \alpha_{2}=\left(E_{1}+E_{2}-E_{1} * E_{2}, I_{1} * I_{2}, N_{1} * N_{2}\right)$,

(2) $\lambda \alpha_{1}=\left(1-\left(1-E_{1}\right)^{\lambda},\left(I_{1}\right)^{\lambda},\left(N_{1}\right)^{\lambda}\right), \lambda \geq 0$.

\section{Pearson's Picture Fuzzy Correlation Coefficient}

The Pearson's correlation coefficient between exact sets can be used to measure the degree of their correlation relationship and depicts whether they are positively or negatively correlated. Up to now, the Pearson's coefficient has been further applied to some fuzzy environments, such as by Szmidt et al. [23], who proposed the correlation coefficient for intuitionistic fuzzy sets, and Chen [24], who introduced the Pearson correlation coefficients between Pythagorean fuzzy and interval-valued Pythagorean fuzzy sets. Meanwhile, Singh [10] proposed several correlation coefficients for PFSs. However, the values of correlation coefficients for PFSs introduced by Singh lie in the interval $[0,1]$, which is inconsistent with traditional correlation coefficients in statistics that lie in range $[-1,1]$. That is to say, the present correlation coefficient only considers positive correlations between PFSs and neglects their negative situation, which reduces the theoretical support and rationality. The coefficient can be defined as follows.

Definition 4 [10]. Let $X$ be a nonempty fixed set, and let $P=\left(p_{1}, p_{2}, \ldots, p_{n}\right)$ and $Q=\left(q_{1}, q_{2}, \ldots, q_{n}\right)$ be two $P F S$ s in $X$. Then, the correlation coefficient between $P$ and $Q$ is defined as

$$
K(P, Q)=\frac{\sum_{i=1}^{n}\left(E_{P_{i}} E_{Q_{i}}+I_{P_{i}} I_{Q_{i}}+N_{P_{i}} N_{Q_{i}}+T_{P_{i}} T_{Q_{i}}\right)}{\left\{\sum_{i=1}^{n}\left(\left(E_{P_{i}}\right)^{2}+\left(I_{P_{i}}\right)^{2}+\left(N_{P_{i}}\right)^{2}+\left(T_{P_{i}}\right)^{2}\right)\right\}^{\frac{1}{2}} \cdot\left\{\sum_{i=1}^{n}\left(\left(E_{Q_{i}}\right)^{2}+\left(I_{Q_{i}}\right)^{2}+\left(N_{Q_{i}}\right)^{2}+\left(T_{Q_{i}}\right)^{2}\right)\right\}^{\frac{1}{2}}}
$$

It is easy to verify that the correlation coefficient $K(P, Q)$ lies in the interval $[0,1]$. Thus, it only depicts positive relationships and not any negative relationships between PFSs. Analogous to the former research, we shall develop a new concept of a picture fuzzy correlation coefficient to form a foundation for the proposed Pearson's picture fuzzy correlation-based approach. The main property of 
this proposed picture fuzzy coefficient is that it lies in the interval $[-1,1]$, which is in accordance with the features of classical methods in statistics. The other feature is that it considers all four elements characterizing the PFSs, thus avoiding any loss of information.

Definition 5. Let $X$ be a nonempty fixed set, and let $P=\left(p_{1}, p_{2}, \ldots, p_{n}\right)$ and $Q=\left(q_{1}, q_{2}, \ldots, q_{n}\right)$ be two PFSs in X. Accordingly, the picture fuzzy correlation coefficient $r(P, Q)$ between $P$ and $Q$ is defined as follows:

$$
r(P, Q)=\frac{1}{4}\left(r_{E}(P, Q)+r_{I}(P, Q)+r_{N}(P, Q)+r_{T}(P, Q)\right)
$$

where

$$
\begin{gathered}
r_{E}(P, Q)=\frac{\sum_{i=1}^{n}\left(E_{p_{i}}-\bar{E}_{p}\right)\left(E_{q_{i}}-\bar{E}_{q}\right)}{\sqrt{\sum_{i=1}^{n}\left(E_{p_{i}}-\bar{E}_{p}\right)^{2}} \sqrt{\sum_{i=1}^{n}\left(E_{q_{i}}-\bar{E}_{q}\right)^{2}}} \\
r_{I}(P, Q)=\frac{\sum_{i=1}^{n}\left(I_{p_{i}}-\bar{I}_{p}\right)\left(I_{q_{i}}-\bar{I}_{q}\right)}{\sqrt{\sum_{i=1}^{n}\left(I_{p_{i}}-\bar{I}_{p}\right)^{2}} \sqrt{\sum_{i=1}^{n}\left(I_{q_{i}}-\bar{I}_{q}\right)^{2}}} \\
r_{N}(P, Q)=\frac{\sum_{i=1}^{n}\left(N_{p_{i}}-\bar{N}_{p}\right)\left(N_{q_{i}}-\bar{N}_{q}\right)}{\sqrt{\sum_{i=1}^{n}\left(N_{p_{i}}-\bar{N}_{p}\right)^{2}} \sqrt{\sum_{i=1}^{n}\left(N_{q_{i}}-\bar{N}_{q}\right)^{2}}} \\
r_{T}(P, Q)=\frac{\sqrt{\sum_{i=1}^{n}\left(T_{p_{i}}-\bar{T}_{p}\right)\left(T_{q_{i}}-\bar{T}_{q}\right)}}{\sqrt{\sum_{i=1}^{n}\left(T_{p_{i}}-\bar{T}_{p}\right)^{2}} \sqrt{\sum_{i=1}^{n}\left(T_{q_{i}}-\bar{T}_{q}\right)^{2}}} .
\end{gathered}
$$

Among Equations (5)-(8), the relevant means of the degrees of positive, neutral, negative, and refusal membership in relation to $p_{i}$ and $q_{i}$ are computed as follows: $\bar{E}_{p}=\sum_{i=1}^{n} E_{p_{i}} / n$, $\bar{E}_{q}=\sum_{i=1}^{n} E_{q_{i}} / n, \bar{I}_{p}=\sum_{i=1}^{n} I_{p_{i}} / n, \bar{I}_{q}=\sum_{i=1}^{n} I_{q_{i}} / n, \bar{N}_{p}=\sum_{i=1}^{n} N_{p_{i}} / n, \bar{N}_{q}=\sum_{i=1}^{n} N_{q_{i}} / n$, $\bar{T}_{p}=\sum_{i=1}^{n} T_{p_{i}} / n$, and $\bar{T}_{q}=\sum_{i=1}^{n} T_{q_{i}} / n$.

Theorem 1. The positive membership component $r_{E}(P, Q)$ in the picture fuzzy correlation coefficient $r(P, Q)$ has the following properties:

(i) $\quad r_{E}(P, Q)=r_{E}(Q, P)$;

(ii) $r_{E}(P, Q)=1$ if $E_{p_{i}}=E_{q_{i}}, \forall i \in[1, n]$;

(iii) $\left|r_{E}(P, Q)\right| \leq 1$.

\section{Proof.}

(i) This is straightforward. 
(ii) If $p_{i}=q_{i}, \forall i \in[1, n]$, it verifies that $\bar{E}_{p}=\bar{E}_{q}$; then we have

$$
r_{E}(P, Q)=\frac{\sum_{i=1}^{n}\left(E_{p_{i}}-\bar{E}_{p}\right)\left(E_{q_{i}}-\bar{E}_{q}\right)}{\sqrt{\sum_{i=1}^{n}\left(E_{p_{i}}-\bar{E}_{p}\right)^{2}} \sqrt{\sum_{i=1}^{n}\left(E_{q_{i}}-\bar{E}_{q}\right)^{2}}}=\frac{\sum_{i=1}^{n}\left(E_{p_{i}}-\bar{E}_{p}\right)^{2}}{\sum_{i=1}^{n}\left(E_{p_{i}}-\bar{E}_{p}\right)^{2}}=1 .
$$

(iii) Because $0 \leq E_{p_{i}}, E_{q_{i}} \leq 1, \forall i \in[1, n]$, it follows that $0 \leq \bar{E}_{p}, \bar{E}_{q} \leq 1$, and therefore, we get $-1 \leq E_{p_{i}}-\bar{E}_{p} \leq 1,-1 \leq E_{q_{i}}-\bar{E}_{q} \leq 1,\left(E_{p_{i}}-\bar{E}_{p}\right)^{2} \leq 1$, and $\left(E_{q_{i}}-\bar{E}_{q}\right)^{2} \leq 1$. Thus, we obtain $-n \leq \sum_{i=1}^{n}\left(E_{p_{i}}-\bar{E}_{p}\right)\left(E_{q_{i}}-\bar{E}_{q}\right) \leq n$ and $\sqrt{\sum_{i=1}^{n}\left(E_{p_{i}}-\bar{E}_{p}\right)^{2}} \sqrt{\sum_{i=1}^{n}\left(E_{q_{i}}-\bar{E}_{q}\right)^{2}} \leq \sqrt{n} \cdot \sqrt{n}=n$. It is thus obviously verified that $-1 \leq r_{E}(P, Q) \leq 1$, which implies that $\left|r_{E}(P, Q)\right| \leq 1$.

Theorems $2-4$ can be obtained by a similar analysis.

Theorem 2. The neutral membership component $r_{I}(P, Q)$ in the picture fuzzy correlation coefficient $r(P, Q)$ has the following properties:

(i) $r_{I}(P, Q)=r_{I}(Q, P)$;

(ii) $r_{I}(P, Q)=1$ if $I_{p_{i}}=I_{q_{i}}, \forall i \in[1, n]$;

(iii) $\left|r_{I}(P, Q)\right| \leq 1$.

Theorem 3. The negative membership component $r_{N}(P, Q)$ in the picture fuzzy correlation coefficient $r(P, Q)$ has the following properties:

(i) $r_{N}(P, Q)=r_{N}(Q, P)$;

(ii) $r_{N}(P, Q)=1$ if $N_{p_{i}}=N_{q_{i}}, \forall i \in[1, n]$;

(iii) $\left|r_{N}(P, Q)\right| \leq 1$.

Theorem 4. The refusal membership component $r_{T}(P, Q)$ in the picture fuzzy correlation coefficient $r(P, Q)$ has the following properties:

(i) $r_{T}(P, Q)=r_{T}(Q, P)$;

(ii) $r_{T}(P, Q)=1$ if $T_{p_{i}}=T_{q_{i}}, \forall i \in[1, n]$;

(iii) $\left|r_{T}(P, Q)\right| \leq 1$.

On the basis of Theorems $1-4$, it is easy to obtain Theorem 5 .

Theorem 5. The picture fuzzy correlation coefficient $r(P, Q)$ between $P$ and $Q$ has the following properties:

(i) $r(P, Q)=r(Q, P)$;

(ii) $r(P, Q)=1$ if $p_{i}=q_{i}, \forall i \in[1, n]$;

(iii) $|r(P, Q)| \leq 1$.

\section{Proof.}

(i) This is trivial.

(ii) If $p_{i}=q_{i}, \forall i \in[1, n]$, it holds that $E_{p_{i}}=E_{q_{i}}, I_{p_{i}}=I_{q_{i}}, N_{p_{i}}=N_{q_{i}}$, and $T_{p_{i}}=T_{q_{i}}$; then we directly obtain $r_{E}(P, Q)=1, r_{I}(P, Q)=1, r_{T}(P, Q)=1$, and $r_{T}(P, Q)=1$ according to Theorems 1-4. Therefore, $r(P, Q)=\frac{1}{4}(1+1+1+1)=1$. 
(iii) This is easy to verify based on the results of Theorems $1-4$.

Sometimes, different importance levels of the elements $x_{i} \in X$ shall be incorporated into the calculation process of the picture fuzzy correlation coefficient in practical applications. In this regard, we formulate the weighted fuzzy correlation coefficient, which is defined as follows.

Definition 6. Let $X$ be a nonempty fixed set and $w_{i}$ the importance weight of $x_{i}$, such that $\sum_{i=1}^{n} w_{i}=1$ and $w_{i} \in[0,1]$. Accordingly, the weighted picture fuzzy correlation coefficient $r^{w}(P, Q)$ between $P$ and $Q$ is defined as follows:

$$
r^{w}(P, Q)=\frac{1}{4}\left(r_{E}^{w}(P, Q)+r_{I}^{w}(P, Q)+r_{N}^{w}(P, Q)+r_{T}^{w}(P, Q)\right)
$$

where

$$
\begin{gathered}
r_{E}^{w}(P, Q)=\frac{\sum_{i=1}^{n} w_{i}\left(E_{p_{i}}-\bar{E}_{p}\right)\left(E_{q_{i}}-\bar{E}_{q}\right)}{\sqrt{\sum_{i=1}^{n} w_{i}\left(E_{p_{i}}-\bar{E}_{p}\right)^{2}} \sqrt{\sum_{i=1}^{n} w_{i}\left(E_{q_{i}}-\bar{E}_{q}\right)^{2}}} \\
r_{I}^{w}(P, Q)=\frac{\sum_{i=1}^{n} w_{i}\left(I_{p_{i}}-\bar{I}_{p}\right)\left(I_{q_{i}}-\bar{I}_{q}\right)}{\sqrt{\sum_{i=1}^{n} w_{i}\left(I_{p_{i}}-\bar{I}_{p}\right)^{2}} \sqrt{\sum_{i=1}^{n} w_{i}\left(I_{q_{i}}-\bar{I}_{q}\right)^{2}}} \\
r_{N}^{w}(P, Q)=\frac{\sum_{i=1}^{n} w_{i}\left(N_{p_{i}}-\bar{N}_{p}\right)\left(N_{q_{i}}-\bar{N}_{q}\right)}{\sqrt{\sum_{i=1}^{n} w_{i}\left(N_{p_{i}}-\bar{N}_{p}\right)^{2}} \sqrt{\sum_{i=1}^{n} w_{i}\left(N_{q_{i}}-\bar{N}_{q}\right)^{2}}} \\
r_{T}^{w}(P, Q)=\frac{\sqrt{\sum_{i=1}^{n} w_{i}\left(T_{p_{i}}-\bar{T}_{p}\right)\left(T_{q_{i}}-\bar{T}_{q}\right)}}{\sqrt{\sum_{i=1}^{n} w_{i}\left(T_{p_{i}}-\bar{T}_{p}\right)^{2}} \sqrt{\sum_{i=1}^{n} w_{i}\left(T_{q_{i}}-\bar{T}_{q}\right)^{2}}}
\end{gathered}
$$

Theorem 6. The weighted picture fuzzy correlation coefficient $r^{w}(P, Q)$ between $P$ and $Q$ has the following important properties:

(i) $r^{w}(P, Q)=r^{w}(Q, P)$;

(ii) $r^{w}(P, Q)=1$ if $p_{i}=q_{i}, \forall i \in[1, n]$;

(iii) $\left|r^{w}(P, Q)\right| \leq 1$;

(iv) $r^{w}(P, Q)=r(P, Q)$ if $w_{i}=i / n, \forall i \in[1, n]$.

\section{Proof.}

(i) This is straightforward.

(ii) If $p_{i}=q_{i}, \forall i \in[1, n]$, it follows that $E_{p}=E_{q}, I_{p}=I_{q}$, and $N_{p}=N_{q}$. Then, it directly follows that $r_{E}^{w}(P, Q)=1, r_{I}^{w}(P, Q)=1, r_{N}^{w}(P, Q)=1$, and $r_{T}^{w}(P, Q)=1$. Therefore, we have $r^{w}(P, Q)=$ $\frac{1}{4}(1+1+1+1)=1$.

(iii) It is easy to prove that $\left|r_{E}^{w}(P, Q)\right| \leq 1,\left|r_{I}^{w}(P, Q)\right| \leq 1,\left|r_{N}^{w}(P, Q)\right| \leq 1$, and $\left|r_{T}^{w}(P, Q)\right| \leq 1$. Thus, it is clearly verified that $\left|r_{E}^{w}(P, Q)\right| \leq 1$. 
(iv) The proof is trivial.

\section{The Pearson's Correlation-Based Model for MADM Involving Picture Fuzzy Information}

The technique for order preference by similarity to ideal solutions (TOPSIS) $[25,26]$ is one of most widely used decision-making methods; its main idea is to choose and determine the best alternative based on the distances between potential candidate schemes and positive and negative ideal solutions. At present, the TOPSIS approach has been broadly studied and extended to many kinds of areas and uncertain environments [27-29]. Recently, instead of using distance measures, Chen [24] proposed a correlation-based TOPSIS method for Pythagorean fuzzy MADM problems. Motivated by Chen's work [24], based on the developed concept of the (weighted) picture fuzzy correlation coefficient, in this paper we provide a novel Pearson's correlation-based TOPSIS method to handle uncertain MADM problems in the picture fuzzy context. Moreover, a correlation-based picture fuzzy closeness index is introduced to rank the potential alternatives.

Consider a MADM problem within the picture fuzzy environment. Let $A=\left\{A_{1}, A_{2}, \ldots, A_{m}\right\}$ denote the discrete set of candidate alternatives, and let $S=\left\{S_{1}, S_{2}, \ldots, S_{n}\right\}$ be a finite set of evaluative attributes. Assume that the weight vector of the attributes is $w^{T}=\left(w_{1}, w_{2}, \ldots, w_{n}\right)^{T}$, meeting $\sum_{j=1}^{n} w_{j}=1$ and $w_{j} \in[0,1]$. The evaluation of alternative $A_{i} \in A$ related to the attribute $S_{j} \in S$ is represented by a PFV $p \prime_{i j}=\left(E{ }^{\prime}{ }_{i j}, I{ }^{\prime}{ }_{i j}, N{ }^{\prime}{ }_{i j}\right)$ such that $E \prime_{i j}, I{ }^{\prime}{ }_{i j}, N \prime_{i j} \in[0,1]$ and $0 \leq E \prime_{i j}+I{ }^{\prime}{ }_{i j}+N \prime_{i j} \leq 1$. Then, the degree of refusal membership for each $p \prime_{i j}$ is calculated as $\pi \prime_{i j}=1-\left(E \prime_{i j}+I{ }_{i j}+N^{\prime}{ }_{i j}\right)$. Accordingly, a picture fuzzy decision matrix can be formed as

$$
R \mathbf{I}=\left(p_{i j}^{\prime}\right)_{m \times n}=\left(\begin{array}{ccc}
p_{11}^{\prime} & \cdots & p_{1 n}^{\prime} \\
\vdots & \ddots & \vdots \\
p_{m 1}^{\prime} & \cdots & p_{m n}^{\prime}
\end{array}\right) .
$$

If all the attributes $S_{j} \in S(j=1,2, \ldots, n)$ belong to the same type, then there is no need to normalize their values. However, benefit attributes (the larger the attribute assessment, the better) and cost attributes (smaller attribute evaluations indicate higher preference) generally appear in MADM problems. In such situations, we convert the cost attribute evaluations into benefit attribute values, then the picture fuzzy decision matrix in Equation (14) can be transformed into the following formula:

$$
R=\left(p_{i j}\right)_{m \times n}=\left(\begin{array}{ccc}
p_{11} & \cdots & p_{1 n} \\
\vdots & \ddots & \vdots \\
p_{m 1} & \cdots & p_{m n}
\end{array}\right),
$$

where

$$
p_{i j}=\left(E_{i j}, I_{i j}, N_{i j}\right)=\left\{\begin{array}{c}
p_{i j}, \text { for benefit attribute } S_{j} \\
\bar{p}{ }_{i j}, \text { for cost attribute } S_{j}
\end{array},\right.
$$

and $\bar{p}{ }_{i j}$ is the complement of $p \prime_{i j}$ such that $\bar{p}{ }_{i j}=\left(N{ }_{i j}, I{ }^{\prime}{ }_{i j}, E \prime_{i j}\right)$.

Furthermore, the PF characteristic $p_{i}$ for alternative $A_{i}$ can be precisely represented as the following vector:

$$
p_{i}=\left\{p_{i 1}, p_{i 2}, \ldots, p_{i n}\right\}=\left\{\left(E_{i 1}, I_{i 1}, N_{i 1}\right),\left(E_{i 2}, I_{i 2}, N_{i 2}\right), \ldots,\left(E_{i n}, I_{i n}, N_{i n}\right)\right\} .
$$

On the basis of the abovementioned information, next we present a novel method based on Pearson-like correlation for addressing picture fuzzy MADM problems. The decision procedure can be summarized as follows. 
Step 1. Establish the decision matrix $R=\left(p_{i j}\right)_{m \times n}$ according to the decision-makers' evaluation information. Also, identify the weighting vector $w^{T}=\left(w_{1}, w_{2}, \ldots, w_{n}\right)^{T}$ with respect to the $n$ attributes.

Step 2. Establish the picture fuzzy positive ideal decision $p^{+}=\left\{p_{1}^{+}, p_{2}^{+}, \ldots, p_{n}^{+}\right\}$and picture fuzzy negative ideal decision $p^{-}=\left\{p_{1}^{-}, p_{2}^{-}, \ldots, p_{n}^{-}\right\}$using Equations (17) and (18):

$$
\begin{aligned}
& p_{j}^{+}=\left(E_{j}^{+}, I_{j}^{+}, N_{j}^{+}\right)=\left(\max _{i}\left\{E_{i j}\right\}, \min _{i}\left\{I_{i j}\right\}, \min _{i}\left\{N_{i j}\right\}\right), \\
& p_{j}^{-}=\left(E_{j}^{-}, I_{j}^{-}, N_{j}^{-}\right)=\left(\min _{i}\left\{E_{i j}\right\}, \min _{i}\left\{I_{i j}\right\}, \max _{i}\left\{N_{i j}\right\}\right) .
\end{aligned}
$$

Moreover, the respective refusal degrees relative to $p_{j}^{+}$and $p_{j}^{-}$can be calculated as follows: $\pi_{j}^{+}=1-\left(E_{j}^{+}+I_{j}^{+}+N_{j}^{+}\right)$and $\pi_{j}^{-}=1-\left(E_{j}^{-}+I_{j}^{-}+N_{j}^{-}\right)$.

Step 3. Utilize Equation (9) to determine the picture fuzzy weighted correlation coefficients $r^{w}\left(p_{i}, p^{+}\right)$and $r^{w}\left(p_{i}, p^{-}\right)$for each $A_{i} \in A$.

Step 4. Use Equation (20) to compute the picture fuzzy weighted correlation-based closeness index $C C^{w}\left(p_{i}\right)$ for all $A_{i} \in A$ :

$$
C C^{w}\left(p_{i}\right)=\frac{1+r^{w}\left(p_{i}, p^{+}\right)}{2+r^{w}\left(p_{i}, p^{+}\right)+r^{w}\left(p_{i}, p^{-}\right)}
$$

Step 5. Sort all the alternatives $A_{i}(i=1,2, \ldots, n)$ by descending $C C^{w}\left(p_{i}\right)$ values. Obviously, the alternative(s) with the greatest value $C C^{w}\left(p_{i}\right)$ can be considered the best candidate alternative(s).

It is noted that the Equation (20) is originally introduced by Chen [24] to rank the alternatives in Pythagorean fuzzy MADM problems, whereas in this paper it is extended by using the presented picture fuzzy weighted correlation coefficients to solve MADM problems within picture fuzzy contexts. Now we analyze the features of the picture fuzzy weighted correlation-based closeness index defined in Equation (20). Generally, a positive $r^{w}\left(p_{i}, p^{+}\right)$indicates that the picture fuzzy vectors $p_{i}$ and $p^{+}$are positively correlated, whereas a negative $r^{w}\left(p_{i}, p^{+}\right)$shows a negative correlation. Thus, the greater $r^{w}\left(p_{i}, p^{+}\right)$is and the smaller $r^{w}\left(p_{i}, p^{-}\right)$is, the better the alternative $A_{i}$ is. However, the alternative $A_{i}$ closest to $p^{+}$cannot yield the one that is farthest from $p^{-}$. The proposed picture fuzzy weighted correlation-based closeness index $C C^{w}\left(p_{i}\right)$ in Equation (20) is useful for addressing this issue because it can calculate the degree to which $p_{i}$ is simultaneously highly associated to $p^{+}$and far from $p^{-}$. Moreover, $C C^{w}\left(p_{i}\right)$ satisfies the condition $0 \leq C C^{w}\left(p_{i}\right) \leq 1$ as $-1 \leq r^{w}\left(p_{i}, p^{+}\right) \leq 1$ and $-1 \leq r^{w}\left(p_{i}, p^{-}\right) \leq 1$.

\section{Numerical Example}

In this section we provide a numerical example for assessing emerging technology commercialization within the picture fuzzy environment to verify the applicability of the developed approach. A MADM problem of selecting emerging technology enterprises is defined by five potential alternatives $A_{i}(i=1,2, \ldots, 5)$ and six evaluative attributes [15]: (1) $S_{1}=$ technical advancement; (2) $S_{2}$ $=$ development of science and technology; (3) $S_{3}=$ industrialization infrastructure; (4) $S_{4}=$ potential market and market risk; (5) $S_{5}=$ employment creation; and (6) $S_{6}=$ financial conditions.

Step 1. To achieve their objective, the experts considered carefully the six evaluative attributes for each alternative and presented the picture fuzzy decision matrix depicted in Table 1.

Table 1. The picture fuzzy decision matrix.

\begin{tabular}{ccccccc}
\hline & $S_{1}$ & $S_{2}$ & $S_{3}$ & $S_{4}$ & $S_{5}$ & $S_{6}$ \\
\hline$A_{1}$ & $(0.53,0.33,0.09)$ & $(0.89,0.08,0.03)$ & $(0.42,0.35,0.18)$ & $(0.08,0.89,0.02)$ & $(0.33,0.51,0.12)$ & $(0.17,0.53,0.13)$ \\
$A_{2}$ & $(0.85,0.09,0.05)$ & $(0.74,0.16,0.10)$ & $(0.02,0.89,0.05)$ & $(0.08,0.84,0.06)$ & $(0.16,0.71,0.05)$ & $(0.15,0.73,0.08)$ \\
$A_{3}$ & $(0.91,0.03,0.02)$ & $(0.07,0.09,0.05)$ & $(0.04,0.85,0.10)$ & $(0.68,0.26,0.06)$ & $(0.15,0.76,0.07)$ & $(0.31,0.39,0.25)$ \\
$A_{4}$ & $(0.73,0,12,0.08)$ & $(0.13,0.64,0.21)$ & $(0.03,0.82,0.13)$ & $(0.73,0.15,0.08)$ & $(0.52,0.31,0.16)$ & $(0.51,0.24,0.21)$ \\
$A_{5}$ & $(0.90,0.05,0.02)$ & $(0.68,0.08,0.21)$ & $(0.05,0.87,0.06)$ & $(0.13,0.75,0.09)$ & $(1.00,0.00,0.00)$ & $(0.91,0.03,0.05)$ \\
\hline
\end{tabular}


Note that all the attributes are of the benefit type, so there was no need to convert them. The weighting vector of the attributes was determined by the experts in advance as $(0.12,0.25,0.09,0.16,0.20,0.18)^{T}$.

Step 2. Using Equations (18) and (19), the picture fuzzy positive ideal decision $p^{+}=\left\{p_{1}^{+}, p_{2}^{+}, \ldots, p_{n}^{+}\right\}$and picture fuzzy negative ideal decision $p^{-}=\left\{p_{1}^{-}, p_{2}^{-}, \ldots, p_{n}^{-}\right\}$were calculated as follows:

$$
\begin{gathered}
p^{+}=\{(0.91,0.05,0.02),(0.89,0.08,0.03),(0.42,0.35,0.05),(0.73,0.15,0.02), \\
(0.52,0.31,0.05),(1.00,0.00,0.00)\} \\
p^{-}=\{(0.53,0.33,0.09),(0.07,0.64,0.21),(0.02,0.89,0.18),(0.08,0.89,0.09), \\
(0.15,0.76,0.16),(0.17,0.53,0.25)\}
\end{gathered}
$$

Taking $p_{1}^{+}$and $p_{2}^{-}$as an example,

$$
\begin{aligned}
p_{1}^{+}=(0.09,0.03,0.02)= & (\max \{0.53,0.73,0.91,0.85,0.90), \min \{0.33,0.12,0.03,0.09,0.05), \\
& \min \{0.09,0.08,0.02,0.05,0.02\}) \\
p_{2}^{-}=(0.07,0.64,0.21)= & (\min \{0.89,0.13,0.07,0.74,0.68), \max \{0.08,0.64,0.09,0.16,0.08\}, \\
& \max \{0.03,0.21,0.05,0.05,0.10,0.21\})
\end{aligned}
$$

Step 3. We computed the picture fuzzy weighted correlation coefficient $r^{w}\left(p_{i}, p^{+}\right)$and $r^{w}\left(p_{i}, p^{-}\right)$ for each $A_{i} \in A$ by means of Equation (9). The calculated results are indicated in Table 2.

Step 4. We used Equation (20) to calculate the index $C C^{w}\left(p_{i}\right)$ for each $A_{i} \in A$. The obtained results are presented in Table 2.

Table 2. Computational results.

\begin{tabular}{cccc}
\hline & $\boldsymbol{r}^{\boldsymbol{w}}\left(\boldsymbol{p}_{\boldsymbol{i}}, \boldsymbol{p}^{+}\right)$ & $\boldsymbol{r}^{\boldsymbol{w}}\left(\boldsymbol{p}_{\boldsymbol{i}}, \boldsymbol{p}^{-}\right)$ & $\boldsymbol{C} C^{\boldsymbol{w}}\left(\boldsymbol{p}_{\boldsymbol{i}}\right)$ \\
\hline$A_{1}$ & 0.0882 & 0.0669 & 0.4640 \\
$A_{2}$ & 0.7505 & 0.1142 & 0.6110 \\
$A_{3}$ & -0.0295 & 0.0766 & 0.3666 \\
$A_{4}$ & 0.0039 & 0.4339 & 0.4118 \\
$A_{5}$ & 0.5854 & 0.0669 & 0.5977 \\
\hline
\end{tabular}

Step 5. From Table 2, we can observe that the picture fuzzy correlation-based closeness index values for each $A_{i} \in A$ are

$$
C C^{w}\left(p_{1}\right)=0.4640, C C^{w}\left(p_{2}\right)=0.6110, C C^{w}\left(p_{3}\right)=0.3666, C C^{w}\left(p_{4}\right)=0.4118, C C^{w}\left(p_{5}\right)=0.5977 .
$$

As explained previously, the larger the $C C^{w}\left(p_{i}\right)$, the better the alternative $A_{i}$. Therefore, the ranking orders of the alternatives are $A_{2}>A_{5}>A_{1}>A_{4}>A_{3}$. Thus, the best choice is $A_{2}$.

To illustrate the effectiveness of the presented Pearson's picture fuzzy correlation-based method, next we provide a comparative analysis with other methods. Firstly, if we merely consider the picture fuzzy positive ideal solution $p^{+}$as the reference point, then the obtained ranking result is $A_{2}>A_{5}>A_{1}>A_{4}>A_{3}$, which is consistent with the presented Pearson's correlation-based methodology. The obtained ranking order is $A_{1}=A_{5}>A_{3}>A_{2}>A_{4}$ if only the picture fuzzy negative ideal solution $p^{-}$is considered. Obviously, different reference points may result in different ranking orders. From this point, one can see that the proposed method can achieve a more scientific and reasonable result as it fully takes into account the positive ideal and negative ideal solutions.

If we construct the correlation-based TOPSIS method by using Singh's correlation coefficient [10] and use it in the calculation and analysis of this example, then we can get the results listed in Table 3. 
From Table 3 , one can see that the ranking orders of the alternatives are $A_{4}>A_{5}>A_{1}>A_{2}>A_{3}$, and the best choice is $A_{4}$. Therefore, a different ranking of the alternatives and a different optimal scheme were produced by the different correlation coefficient used. In fact, our correlation-based TOPSIS model employing the new correlation coefficient is more effective and reasonable than the existing method.

Wei et al. [15] proposed a picture fuzzy projection concept to solve the same question presented in this paper, in which the projection $\operatorname{Prj}_{A^{+}}\left(A_{i}\right)$ between the alternative $A_{i}$ and the idea point $A^{+}$was applied to assess the potential candidates. The computational results based on this projection model are

$$
\operatorname{Prj}_{A^{+}}\left(A_{1}\right)=0.235, \operatorname{Prj}_{A^{+}}\left(A_{2}\right)=0.297, \operatorname{Prj}_{A^{+}}\left(A_{3}\right)=0.156, \operatorname{Prj}_{A^{+}}\left(A_{4}\right)=0.194, \operatorname{Prj}_{A^{+}}\left(A_{5}\right)=0.283
$$

Therefore, the sorting order according to the descending values of $\operatorname{Prj}_{A^{+}}\left(A_{i}\right)$ is $A_{2}>A_{5}>A_{1}>$ $A_{4}>A_{3}$, which renders the same ranking as that obtained by our presented Pearson's correlation-based method. By the comparative discussions, one can see that the developed methodology yields a reasonable result for experts to address uncertain MADM problems in a picture fuzzy environment.

Table 3. Results rendered by the correlation-based technique for order preference by similarity to ideal solutions (TOPSIS) based on Singh's correlation coefficient.

\begin{tabular}{cccc}
\hline & $\boldsymbol{r}^{\boldsymbol{w}}\left(\boldsymbol{p}_{\boldsymbol{i}}, \boldsymbol{p}^{+}\right)$ & $\boldsymbol{r}^{\boldsymbol{w}}\left(\boldsymbol{p}_{\boldsymbol{i}}, \boldsymbol{p}^{-}\right)$ & $\boldsymbol{C} C^{\boldsymbol{w}}\left(\boldsymbol{p}_{\boldsymbol{i}}\right)$ \\
\hline$A_{1}$ & 0.6863 & 0.3672 & 0.5523 \\
$A_{2}$ & 0.7161 & 0.4426 & 0.5433 \\
$A_{3}$ & 0.5576 & 0.6452 & 0.4863 \\
$A_{4}$ & 0.8159 & 0.4045 & 0.5639 \\
$A_{5}$ & 0.8184 & 0.4403 & 0.5580 \\
\hline
\end{tabular}

\section{Conclusions}

With MADM problems growing more and more complicated under uncertain environments, the conception of the picture fuzzy set equipped decision-makers with an effective method to portray a high degree of vagueness and ambiguity during multiple attribute assessing processes. This study presented a novel decision-making model using an effective and simple picture fuzzy approach to address picture fuzzy MADM problems in uncertain environments. More specifically, the classic Pearson's correlation coefficient was applied to picture fuzzy contexts. For doing so, a new correlation index formula for PFSs was presented to overcome the flaws in the existing method; the main feature of the new index is that it lies in the interval $[-1,1]$. Moreover, a weighted Pearson's picture fuzzy correlation coefficient was presented to establish the central structure of the developed methodology. Some important properties possessed by these coefficients were discussed and investigated. Furthermore, a weighted correlation-based picture fuzzy closeness index was developed to portray a certain degree of the correlations with the picture fuzzy positive and negative ideal points. A novel Pearson's picture fuzzy correlation-based model was further constructed for addressing uncertain MADM problems involving picture fuzzy evaluation. The usefulness of the proposed methodology was validated through a numerical example and comparative discussions.

In our future work, we expect to study its application in other decision-making areas, such as academical evaluation and credit risk assessment. Further extensions and applications of this approach by considering more general formulations such as induced variables and probability will also be considered.

Author Contributions: Conceptualization, Y.J. and H.W.; writing—original draft preparation, D.S.; validation, S.Z.; writing-review and editing, D.L.; formal analysis, B.P.

Funding: This paper was supported by Major Humanities and Social Sciences Research Projects in Zhejiang Universities (no. 2018QN058), National Natural Science Foundation of China (no. 71671165; no. 71761027), China Postdoctoral Science Foundation (no. 2018QN058), Zhejiang Province Science and Technology Plan Project (no. LGG18F020001; no. LGF19F020008), and Ningbo Science and Technology Project (no. 2019C50008). 
Conflicts of Interest: The authors declare that there is no conflict of interest regarding the publication of this paper.

\section{References}

1. Merigo, J.M.; Zhou, L.; Yu, D.; Alrajeh, N.; Alnowibet, K. Probabilistic OWA Distances Applied to Asset Management. Soft Comput. 2018, 22, 4855-4878. [CrossRef]

2. Yu, L.; Zeng, S.; Merigo, J.M.; Zhang, C. A New Distance Measure Based on the Weighted Induced Method and its Application to Pythagorean Fuzzy Multiple Attribute Group Decision Making. Int. J. Intell. Syst. 2019, 34, 1440-1454. [CrossRef]

3. Rapoport, A.; Wallsten, T.S.; Cox, J.A. Direct and Indirect Scaling of Membership Functions of Probability Phrases. Math. Model. 1987, 9, 397-417. [CrossRef]

4. Smarandache, F. Neutrosophic Set-A Generalization of the Intuitionistic Fuzzy Set. Int. J. Pure Appl. Math. 2005, 24, 287.

5. Yager, R.R. Pythagorean Membership Grades in Multicriteria Decision Making. IEEE Trans. Fuzzy Syst. 2013, 22, 958-965. [CrossRef]

6. Cuong, B.C.; Kreinovich, V. Picture Fuzzy Sets-A New Concept for Computational Intelligence Problems. In Proceedings of the 2013 Third World Congress on Information and Communication Technologies (WICT 2013), Hanoi, Vietnam, 15-18 December 2013; Institute of Electrical and Electronics Engineers (IEEE): Piscataway, NJ, USA, 2013; pp. 1-6.

7. Zadeh, L.A. Fuzzy Sets. Inf. Control. 1965, 8, 338-353. [CrossRef]

8. Atanassov, K.T. Intuitionistic Fuzzy Sets. Fuzzy Sets Syst. 1986, 20, 87-96. [CrossRef]

9. Cuong, B.C. Picture Fuzzy Sets. J. Comput. Sci. Cybern. 2014, 30, 409-420.

10. Singh, P. Correlation Coefficients for Picture Fuzzy Sets. J. Intell. Fuzzy Syst. 2015, 28, 591-604.

11. Garg, H. Some Picture Fuzzy Aggregation Operators and Their Applications to Multicriteria Decision-Making. Arab. J. Sci. Eng. 2017, 42, 5275-5290. [CrossRef]

12. Son Le, H. Measuring Analogousness in Picture Fuzzy Sets: From Picture Distance Measures to Picture Association Measures. Fuzzy Optim. Decis. Mak. 2017, 16, 1-20. [CrossRef]

13. Wei, G.W. Picture Fuzzy Aggregation Operators and their Application to Multiple Attribute Decision Making. J. Intell. Fuzzy Syst. 2017, 33, 713-724. [CrossRef]

14. Wei, G.W. Picture Fuzzy Hamacher Aggregation Operators and their Application to Multiple Attribute Decision Making. Fundam. Inform. 2018, 157, 271-320. [CrossRef]

15. Wei, G.W.; Alsaadi, F.E.; Hayat, T.; Alsaedi, A. Projection Models for Multiple Attribute Decision Making with Picture Fuzzy Information. Int. J. Mach. Learn. Cybern. 2018, 9, 713-719. [CrossRef]

16. Wang, L.; Zhang, H.-Y.; Wang, J.Q.; Li, L. Picture Fuzzy Normalized Projection-Based VIKOR Method for the Risk Evaluation of Construction Project. Appl. Soft Comput. 2018, 64, 216-226. [CrossRef]

17. Zhang, X.Y.; Wang, X.K.; Yu, S.M.; Wang, J.Q.; Wang, T.L. Location Selection of Offshore Wind Power Station by Consensus Decision Framework Using Picture Fuzzymodelling. J. Clean. Prod. 2018, 202, 980-992. [CrossRef]

18. Jana, C.; Senapati, T.; Pal, M.; Yager, R.R. Picture Fuzzy Dombi Aggregation Operators: Application to MADM Process. Appl. Soft Comput. 2019, 74, 99-109. [CrossRef]

19. Ashraf, S.; Mahmood, T.; Abdullah, S.; Khan, Q. Different Approaches to Multi-Criteria Group Decision Making Problems for Picture Fuzzy Environment. Bull. Braz. Math. Soc. 2019, 50, 373-397. [CrossRef]

20. Zeng, S.; Asharf, S.; Arif, M.; Abdullah, S. Application of Exponential Jensen Picture Fuzzy Divergence Measure in Multi-Criteria Group Decision Making. Math 2019, 7, 191. [CrossRef]

21. Liu, P.; You, X. Bidirectional Projection Measure of Linguistic Neutrosophic Numbers and their Application to Multi-Criteria Group Decision Making. Comput. Ind. Eng. 2020, 128, 447-457. [CrossRef]

22. Zhang, L.; Zhan, J.; Alcantud, J.C.R. Novel Classes of Fuzzy Soft $\beta$-Coverings-Based Fuzzy Rough Sets with Applications to Multi-Criteria Fuzzy Group Decision Making. Soft Comput. 2019, 23, 5327-5351. [CrossRef]

23. Szmidt, E.; Kacprzyk, J. Correlation of Intuitionistic Fuzzy Sets. In Proceedings of the Fuzzy Logic and Soft Computing Applications, Cancun, Mexico, 18-21 June 2007; Springer Science and Business Media LLC: Berlin, Germany, 2010; Volume 6178, pp. 169-177.

24. Chen, T.Y. Multiple Criteria Decision Analysis under Complex Uncertainty: A Pearson-Like Correlation-Based Pythagorean Fuzzy Compromise Approach. Int. J. Intell. Syst. 2019, 34, 114-151. [CrossRef] 
25. Yoon, K. Systems Selection by Multiple Attribute Decision Making. Ph.D. Dissertation, Kansas State University, Manhattan, AR, USA, 1980.

26. Hwang, C.L.; Yoon, K. Multiple Attribute Decision Making: Methods and Applications. In A State-of-the-Art Survey; Springer-Verlag: Berlin, Germany, 1981.

27. Zavadskas, E.K.; Mardani, A.; Turskis, Z.; Jusoh, A.; Nor, K.M. Development of TOPSIS Method to Solve Complicated Decision-Making Problems: An Overview on Developments From 2000 to 2015. Int. J. Inf. Technol. Decis. Mak. 2016, 15, 645-682. [CrossRef]

28. Aouadni, S.; Rebai, A.; Turskis, Z. The Meaningful Mixed Data TOPSIS (TOPSIS-MMD) Method and its Application in Supplier Selection. Stud. Inform. Control 2017, 26, 353-363. [CrossRef]

29. Zeng, S.Z.; Chen, S.M.; Fan, K.Y. Interval-Valued Intuitionistic Fuzzy Multiple Attribute Decision Making Based on Nonlinear Programming Methodology and TOPSIS Method. Inf. Sci. 2020, 506, 424-442. [CrossRef]

(C) 2019 by the authors. Licensee MDPI, Basel, Switzerland. This article is an open access article distributed under the terms and conditions of the Creative Commons Attribution (CC BY) license (http://creativecommons.org/licenses/by/4.0/). 